\title{
Integral Representations of Cooperative Game with Fuzzy Coalitions
}

\author{
Jinhui Pang ${ }^{1}$ and Xiang Chen ${ }^{2}$ \\ ${ }^{1}$ Library, Beijing Institute of Technology, Beijing 100081, China \\ ${ }^{2}$ Patent Examination Cooperation Center of the Patent Office, State Intellectual Property Office, Beijing 100088, China \\ Correspondence should be addressed to Jinhui Pang; pangjinhui69@126.com
}

Received 1 December 2013; Accepted 2 January 2014; Published 2 March 2014

Academic Editor: Pu-yan Nie

Copyright (c) 2014 J. Pang and X. Chen. This is an open access article distributed under the Creative Commons Attribution License, which permits unrestricted use, distribution, and reproduction in any medium, provided the original work is properly cited.

\begin{abstract}
Classical extensions of fuzzy game models are based on various integrals, such as Butnariu game and Tsurumi game. A new class of symmetric extension of fuzzy game with fuzzy coalition variables is put forward with Concave integral, where players' expected values are on a partial set of coalitions. Some representations and properties of some limited models are compared in this paper. The explicit formula of characteristic function determined by coalition variables is given. Moreover, a calculation approach of imputations is discussed in detail. The new game could be regarded as a general form of cooperative game. Furthermore, the fuzzy game introduced by Tsurumi is a special case of the proposed game when game is convex.
\end{abstract}

\section{Introduction}

Cooperation in game is often full of vagueness because of players' partial participation and players' preference to some coalitions. Games without precise information have been investigated by stochastic framework $[1,2]$. Generally speaking, probability distributions on game events cannot be obtained perfectly. It is reasonable to adopt fuzzy mathematics to model fuzzy games [3-5].

The main ways of describing cooperative game with fuzzy coalitions aim to extend crisp game theory, which is to construct a one-to-one correspondence between a crisp game and a fuzzy game. At present, there are three views of fuzzy games including games with fuzzy payoffs, games with fuzzy coalitions, and games with both fuzzy payoffs and fuzzy coalitions. Aubin [6] firstly studied games with fuzzy coalitions and proposed a line fuzzy game, and a review of this line fuzzy game can be found in Branzei et al. [7]. Butnariu [8] extended the domain of fuzzy coalitions and defined a fuzzy game. Tsurumi et al. [9] introduced a class of fuzzy games with Choquet integral. In addition, Branzei et al. [7] have done lots of work to model fuzzy cooperative game, which was mainly defined by the associated crisp game corresponding to fuzzy game. Borkotokey [10] investigated a class of cooperative games with fuzzy coalitions and fuzzy characteristic function simultaneously. He proposed another class of fuzzy games different from that defined by Butnariu and Branzei, and so forth, where the characteristic value mapping the set of real numbers to the closed interval $[0,1]$ was of fuzzy quantity.

No matter what game model it is, the representation of characteristic function of games with fuzzy coalitions is an expression of integral, such as linear integral in Butnariu game and Choquet integral in Tsurumi et al. game. In this paper, several integral representations which described the fuzzy characteristic function of the cooperative game will be listed and compared mutually. It has been proved that the Concave integral is concave with respect to fuzzy capacities, which might be interpreted as uncertainty aversion [11]. Consequently, it is a better way to model games with fuzzy coalition variables by Concave integral. We extend characteristic function in fuzzy game on a partial set of coalitions via its decompositions of corresponding crisp coalitions. The classical model is a special case of our approach.

In Section 2, we recall some basic concepts of crisp games and fuzzy games. Integral representations for several games with fuzzy coalitions are introduced in Section 3. Some properties and relationships of the several games will also be discussed in detail. We show the differences among these games by numerical examples. Section 4 studies an important case of game in which players' expected values are on a partial 
set of coalitions. A new class of games which depends on decompositions of coalitions will be proposed by Concave integral. In particular, the so-called game with Choquet integral form is a special case of game with Concave integral form. Moreover, in Section 5, an imputation of the new game is investigated and a calculation approach of imputations is proposed. Finally, some conclusions appear in Section 6.

\section{Crisp Cooperative Game and Fuzzy Cooperative Game}

A crisp cooperative game $v$ on player set $N$ is the characteristic function $v: P(N) \rightarrow R_{+} \cup\{0\}$ with $v(\phi)=0$ and $v(S)(S \in P(N))$ is the worth of coalition $S$. The class of crisp games with player set $N$ is denoted by $G(N, v)$.

The game $v \in G(N, v)$ is convex if $v(S \cup T)+v(S \cap T) \geq$ $v(S)+v(T), \forall S, T \in P(N) . \quad v$ is superadditive if $v(S \cup T) \geq$ $v(S)+v(T), \forall S, T \in P(N), S \cap T=\phi$. Denote all the superadditve crisp cooperative games by $G_{0}(N, v)$.

For a nonempty set $S \in P(N)$, the unanimity games $u_{S}$ are defined by

$$
u_{S}(T)= \begin{cases}1, & \text { if } S \subseteq T, \\ 0, & \text { otherwise }\end{cases}
$$

Each cooperative game $v \in G(N, v)$ can be represented by $u_{S}$ as follows:

$$
v=\sum_{T \in P(N) \backslash\{\phi\}} c_{S}(v) u_{S},
$$

where $c_{S}(v)=\sum_{T \in P(N): T \subseteq S}(-1)^{|S|-|T|} v(T)$.

Definition 1. An imputation for a crisp cooperative game $v \in$ $G(N, v)$ is a vector $x=\left(x_{1}, x_{2}, \ldots, x_{n}\right) \in R_{+}^{n} \cup\{0\}$ satisfying

(1) $\sum_{i \in N} x_{i}=v(N)$,

(2) $x_{i} \geq v(\{i\}), \forall i \in N$.

Denote all of imputations for a crisp cooperative game $v \in G(N, v)$ as a set $I(v)(N)$. When $v \in G_{0}(N, v), I(v)(N)$ is nonempty.

The Shapley value of player $i$ has a unique expression given by

$$
\operatorname{Sh}_{i}(v)=\sum_{T \subseteq N \backslash i} \frac{(|N|-|T|-1)|T| !}{|N| !}[v(T \cup i)-v(T)],
$$

where $|\cdot|$ is the cardinality of a coalition.

When $v \in G_{0}(N, v)$, the Shapley vector $\operatorname{Sh}(v)=$ $\left(\mathrm{Sh}_{1}(v), \mathrm{Sh}_{2}(v), \ldots, \mathrm{Sh}_{n}(v)\right) \in I(v)(N)$.

We call $\widetilde{S}$ a fuzzy coalition with coalition variable $s=$ $\left(s_{1}, s_{2}, \ldots, s_{n}\right)$, where $s_{i} \in[0,1]$ is a constant participation level of player $i$. The set of fuzzy coalitions in $N$ is denoted by $L(N)$. If $\widetilde{S}$ is a fuzzy subset of $N$, its support is defined by $\operatorname{Supp}(\widetilde{S})=\left\{i \in N \mid s_{i}>0\right\}$ and its level subset is denoted by $\widetilde{S}$ as $[\widetilde{S}]_{r}=\left\{i \in N \mid s_{i} \geq r, r \in[0,1]\right\}$. If $\widetilde{S} \subseteq \widetilde{U}$, it means that $s_{i} \leq u_{i}$ for any $i \in N$. The identical level set $\widetilde{S}_{r}=\left\{i \in N, s_{i}=r, r \in[0,1]\right\}$ is the players set with the same participation level $r$.
A characteristic function of cooperative game is a function $v: L(N) \rightarrow R$ such that $v(\phi)=0$. The real value function $v$ associates to each coalition $\widetilde{S}$ with worth $v(\widetilde{S})$, which measures the utility of forming coalition $\widetilde{S}$. We take the notation $v \in \widetilde{G}$ as a fuzzy game on $N$ with the characteristic function $v$.

For any fuzzy coalition $\widetilde{S}, \widetilde{U} \in L(N), \widetilde{S} \vee \widetilde{U}$ is the union of two fuzzy coalitions $\widetilde{S}$ and $\widetilde{U}$, and when $i \in \operatorname{Supp} \widetilde{S} \cup \operatorname{Supp} \widetilde{U}$, the coalition variable $(\widetilde{S} \vee \widetilde{U})(i)=s_{i} \vee u_{i}$ and otherwise $(\widetilde{S} \vee$ $\widetilde{U})(i)=0$.

Similarly, $\widetilde{S} \wedge \widetilde{U}$ is the intersection of two fuzzy coalitions $\widetilde{S}$ and $\widetilde{U}$ with the coalition variable $(\widetilde{S} \wedge \widetilde{U})(i)=s_{i} \wedge u_{i}$ for any $i \in \operatorname{Supp} \widetilde{S} \cap \operatorname{Supp} \widetilde{U}$.

Let $v \in \widetilde{G}$; the game $v$ is superadditive if $v(\widetilde{S} \vee \widetilde{U}) \geq v(\widetilde{S})+$ $v(\widetilde{U})$ for all $\widetilde{S}, \widetilde{U} \in L(N)$ and $\widetilde{S} \wedge \widetilde{U}=\phi$. The game $v$ is fuzzy convex if $v(\widetilde{S})+v(\widetilde{U}) \leq v(\widetilde{S} \vee \widetilde{U})+v(\widetilde{S} \wedge \widetilde{U}) . \widetilde{S} \in L(N)$ is a fuzzy carrier in fuzzy coalition $\widetilde{U}$ if $v(\widetilde{S} \wedge \widetilde{T})=v(\widetilde{T})$, for all $\widetilde{T} \subseteq \widetilde{U}$.

Let $N$ be a nonempty set and let $P(N)$ be the power set of $N$. The function $\mu: p(N) \rightarrow R^{+}$is called a capacity on $N$ if $\mu(\phi)=0$ and $\mu(A) \leq \mu(B)$ whenever $A \subset B$, where $\mu(i)$ indicates the weight of the elements $i \in N(i=1,2, \ldots, n)$.

Next, we will give Choquet integral [12] and Concave integral [11].

Definition 2. Let $\mu$ be a capacity on $N$; the Choquet integral of a nonnegative function $f: N \rightarrow R^{+}$with respect to $\mu$ is defined by

$$
\text { (C) } \int_{N} f d \mu=\int^{C h} f d \mu=\int_{0}^{\infty} \mu\left(\left\{i \in N \mid f_{i} \geq r\right\}\right) d r \text {, }
$$

where $f_{i}=f(\{i\})$.

The Choquet integral with a discrete capacity $\mu$ can be rewritten as follows:

$$
\begin{aligned}
G(N) & =(C) \int_{N} f d \mu \\
& =\sum_{i=2}^{n}\left(f_{(i)}-f_{(i-1)}\right) \mu(\{(i),(i+1), \ldots,(n)\})+f_{(1)},
\end{aligned}
$$

where $(\cdot)$ indicates a permutation of $N$ such that $f_{(1)} \leq f_{(2)} \leq$ $\cdots \leq f_{(n)}$.

Definition 3. Let $\mu$ be a capacity on $N$. Fix a nonnegative random variable $X$, and define

$$
\int^{\mathrm{Cav}} X d u=\min \{f(X)\}
$$

where the minimum is taken over all concave and homogeneous functions $f: R_{+}^{n} \rightarrow R$ satisfying $f\left(1_{R}\right) \geq \mu(R)$ for every $R \subseteq N$ and $1_{R}$ is an indicator of $R \subseteq N$.

It has been proved that $\int^{\mathrm{Ch}} X d \mu=\int^{\mathrm{Cav}} X d \mu$ if and only if $\mu$ is convex (see [13]). 


\section{Integral Representations for Some Limited Games with Fuzzy Coalitions}

Butnariu [14] defined a characteristic function of cooperative game with proportional values which was weighted by participation level set.

Definition 4. The game $v \in \widetilde{G}$ is said to be a game with proportional values if and only if

$$
v(\widetilde{S})=\sum_{r \in[0,1]} v\left(\widetilde{S}_{r}\right) \cdot r, \quad \forall \widetilde{S} \in L(N)
$$

The fuzzy game with proportional values corresponds to a crisp game as the associated fuzzy game. For the sake of simplicity, we will denote the fuzzy game with proportional values as $\widetilde{G}^{P}$.

Example 5. Let $N=\{1,2,3,4\}$ and let $v$ be a characteristic function on $N$ which is an output of joint workers; values on crisp coalitions are listed in Table 1.

Consider the fuzzy game $v(\widetilde{U}) \in \widetilde{G}^{P}$ and the fuzzy coalition $\widetilde{U}$ corresponding to coalition variable $u=(0.4,0.4,0.6$, $0.7)$, by Definition 4 ,

$$
\begin{aligned}
v(\widetilde{U})= & \sum_{r \in[0,1]} v\left(\widetilde{U}_{r}\right) \cdot r=0.4 \times v(\{1,2,3,4\}) \\
& +0.6 v(\{3,4\})+0.7 v(\{4\})=87.2 .
\end{aligned}
$$

Butnariu and Kroupa [15] proposed a class of games with weight function which was extension of games with proportional value.

Definition 6. The game $v: L(N) \rightarrow R$ satisfying $v(\phi)=0$ is called a fuzzy game with weight function if and only if

$$
v(\widetilde{S})=\sum_{r \in[0,1]} \psi(r) v\left(\widetilde{S}_{r}\right)
$$

where $\psi:[0,1] \rightarrow R$ is a function with the properties $\psi(r)=$ $0 \Leftrightarrow r=0$ and $\psi(1)=1$.

The set of games with weight functions is denoted by $\widetilde{G}^{\psi}$. If $\psi(r)=r$; then the fuzzy game $v \in \widetilde{G}^{\psi}$ is equivalent to the game $v \in \widetilde{G}^{P}$.

Example 7. Let the game be the same as Example 5, and suppose players set the weight function $\psi(t)=t^{3}-t^{2}+t$, $t \in[0,1]$; then $v(\widetilde{U}) \in \widetilde{G}^{\psi}, \psi(0.4)=0.304, \psi(0.6)=0.456$, $\psi(0.7)=0.553$, and

$$
\begin{aligned}
v(\widetilde{U})= & \sum_{t \in[0,1]} \psi(t) v\left(\widetilde{U}_{t}\right) \\
= & \psi(0.4) \times v(\{1,2,3,4\})+\psi(0.6) v(\{3,4\}) \\
& +\psi(0.7) v(\{4\})=66.608
\end{aligned}
$$

From Example 7, we know that the game value is much greater to depend on the weight function. In spite of the fact
TABLE 1: Values on crisp coalitions.

\begin{tabular}{lccccccc}
\hline$S$ & $v(S)$ & $S$ & $v(S)$ & $S$ & $v(S)$ & $S$ & $v(S)$ \\
\hline 11$\}$ & 13 & $\{1,2\}$ & 32 & $\{2,4\}$ & 38 & $\{1,3,4\}$ & 80 \\
$\{2\}$ & 12 & $\{1,3\}$ & 38 & $\{3,4\}$ & 40 & $\{2,3,4\}$ & 80 \\
$\{3\}$ & 11 & $\{1,4\}$ & 38 & $\{1,2,3\}$ & 50 & $\{1,2,3,4\}$ & 130 \\
$\{4\}$ & 16 & $\{2,3\}$ & 38 & $\{1,2,4\}$ & 45 & & \\
\hline
\end{tabular}

that crisp games are often considered to be monotone nondecreasing, Tsurumi et al. thought that most of this class of games are neither monotone nondecreasing nor continuous with regard to rates of players' participation. They introduced a class of fuzzy games, simply denoted by $\widetilde{G}^{C}$ as follows.

Definition 8. Given $\widetilde{S} \in L(N)$, let $Q(\widetilde{S})=\left\{s_{i} \mid s_{i}>0, i \in N\right\}$ and let $q(\widetilde{S})$ be the cardinality of $Q(\widetilde{S})$. The elements in $Q(\widetilde{S})$ are rewritten by the increasing order as $h_{1}<h_{2}<\cdots<h_{q(\widetilde{S})}$. Then a game $v: L(N) \rightarrow R$ is said to be a fuzzy game with Choquet integral form if and only if the following holds:

$$
v(\widetilde{S})=\sum_{l=1}^{q(\widetilde{S})} v\left([\widetilde{S}]_{h_{l}}\right) \cdot\left(h_{l}-h_{l-1}\right),
$$

for any $\widetilde{S} \in L(N)$, where $h_{0}=0$.

It is apparent that Choquet integral [12] is an integral form for a general class of fuzzy measures, so the fuzzy game model proposed by Tsurumi is Choquet integral of the function $h$ with respect to $v$ derived from level set. Because the case that $h_{l-1}<h_{l}$ implies $[\widetilde{S}]_{h_{l}} \subseteq[\widetilde{S}]_{h_{l-1}}$, so the worth of coalition $\widetilde{S}$ is the maximum sum on all subsets which make an including chain.

Example 9. Take the same game as Example 5; rearrange the fuzzy coalition variable $u=(0.4,0.4,0.6,0.7)$ as $0.4<0.6<$ 0.7 ; by (11), we get the game value $v(\widetilde{U}) \in \widetilde{G}^{C}$ :

$$
\begin{aligned}
v(\widetilde{U})= & \sum_{l=1}^{q(\widetilde{S})} v\left([\widetilde{U}]_{h_{l}}\right) \cdot\left(h_{l}-h_{l-1}\right) \\
= & v\left([\widetilde{U}]_{0.4}\right) \times 0.4+v\left([\widetilde{U}]_{0.6}\right) \\
& \times(0.6-0.4)+v\left([\widetilde{U}]_{0.7}\right) \times(0.7-0.6) \\
= & v(\{1,2,3,4\}) \times 0.4+v(\{3,4\}) \times 0.2+v(\{4\}) \times 0.1 \\
= & 61.6 .
\end{aligned}
$$

A fuzzy game with Choquet integral form has the following properties [9].

Proposition 10. Let $v \in \widetilde{G}^{C}$, for any $\widetilde{K}, \widetilde{U} \in L(N)$ and $\widetilde{K} \subseteq \widetilde{U}$; then the following holds:

$$
v(\widetilde{K}) \leq v(\widetilde{U}) .
$$

Proposition 11. Let $v \in \widetilde{G}^{C}$; define the distance $H(\widetilde{K}, \widetilde{U})=$ $\max _{i \in N}\left|k_{i}-u_{i}\right|$ for any $\widetilde{K}, \widetilde{U} \in L(N)$; then $v$ is continuous. 
Proposition 12. Let $v \in \widetilde{G}^{C}$ and $\widetilde{S}, \widetilde{T} \in L(N)$ such that $v(\widetilde{S})=$ $v(\widetilde{T})$ if and only if

$$
v\left([\widetilde{S}]_{h}\right)=v\left([\widetilde{T}]_{h}\right) \quad \text { for any } h \in(0,1] .
$$

Borkotokey [10] defined the following extended class of fuzzy games, when both coalitions and expectation are fuzzy.

Definition 13. Let $v \in \widetilde{G}^{C}$ and let $\delta>0$ be a real number, for any a fuzzy coalition $\widetilde{S} \in L(N)$; define a function $w^{\delta}$ : $L(N)^{R^{+} \cup\{0\}} \rightarrow L\left(R^{+} \cup\{0\}\right)^{[0,1]}$ satisfying

(i) if $x \neq v(\widetilde{S})$, then $w^{\delta}(\widetilde{K})(x)=\vee\left\{\bigvee_{i \in N} k_{i}\right\}$ where $\widetilde{K} \epsilon$ $L(N), v(\widetilde{K}) \in[v(\widetilde{S})-\delta, v(\widetilde{S})]$ and $|v(\widetilde{K})-x| \leq \delta$,

(ii) if $x=v(\widetilde{S})$, then $w^{\delta}(\widetilde{K})(x)=1$.

We note that this game $w^{\delta}(\widetilde{K}), \forall \widetilde{K} \in L(N)$ is an extended fuzzy game with fuzzy coalitions and vague expectation. For a sufficiently small $\delta>0, w^{\delta}(\widetilde{S})$ is continuous for every fuzzy coalition $\widetilde{S} \in L(N)$. The corresponding class of such games is denoted by $\widetilde{G}^{\delta}$.

Example 14. We continue to consider Example 9; for any $\delta>0$ and $\widetilde{K} \in L(N)$ with fuzzy coalition variable $k=$ $\left(k_{1}, k_{2}, k_{3}, k_{4}\right)$, let $v(\widetilde{K}) \in \widetilde{G}^{\delta}$; then

$$
w^{\delta}(\widetilde{K})(x)= \begin{cases}\vee\left\{\vee_{i \in N} k_{i}\right\}, & \text { if } 61.6-\delta<x<61.6 \\ 1, & \text { if } 61.6<x<61.6+\delta \\ 0, & \text { otherwise. }\end{cases}
$$

In game $v \in \widetilde{G}^{\delta}$, when players' expected values are among $v(\widetilde{S})-\delta$ and $v(\widetilde{S})$, they will set their maximum contribution level to the fuzzy coalition $\widetilde{K} \in L(N)$. By contrast, the maximum contribution of $\widetilde{K} \in L(N)$ keeps $x$ sufficiently close to $v(\widetilde{K}) \in \widetilde{G}^{\delta}$.

Proposition 15. Let $v \in \widetilde{G}^{C}$; then $w^{\delta}$ is monotone nondecreasing when the game $v$ is monotone nondecreasing. That is, for every pair of fuzzy coalitions $\widetilde{S}$ and $\widetilde{T}$ such that $\widetilde{T} \subseteq \widetilde{S}$ it would imply

$$
w^{\delta}(\widetilde{T}) \leq w^{\delta}(\widetilde{S}) .
$$

Proposition 16. Let $v \in \widetilde{G}^{C}$; then the game $w^{\delta}$ is

(1) superadditive if

$$
w^{\delta}(\widetilde{S} \vee \widetilde{T}) \geq w^{\delta}(\widetilde{S})+w^{\delta}(\widetilde{T}),
$$

when $w^{\delta}(\widetilde{S} \vee \widetilde{T})=0$ and $v \in \widetilde{G}^{C}$ is superadditive,

(2) convex if

$$
w^{\delta}(\widetilde{S} \vee \widetilde{T})+w^{\delta}(\widetilde{S} \wedge \widetilde{T}) \geq w^{\delta}(\widetilde{S})+w^{\delta}(\widetilde{T}),
$$

when $v \in \widetilde{G}^{C}$ is convex.

\section{A New Fuzzy Games with Concave Integral}

As mentioned above, the present forms for game with fuzzy coalitions are only limited to some special games. Next, we will consider another extended game with fuzzy coalitions, that is, the fuzzy game with Concave integral, where Tsurumi game can be taken as a special case for the proposed new game. Firstly, we recall the fuzzy capacity and the Concave integral.

Definition 17. Let $\widetilde{A} \in L(N)$; the pair $(v, \widetilde{A})$ is said to be a fuzzy capacity game if $v: L(N) \rightarrow R^{+} \cup\{0\}$ is monotonic, continuous, and there is a positive $M$ such that $v(a) \leq M|a|$ for every $a \in L(\widetilde{A})$.

Definition 18. Let $\widetilde{A} \in L(N)$; the pair $(v, \widetilde{A})$ is said to be an additive fuzzy capacity game, for every fuzzy coalition variable $T \in L(\widetilde{A})$, if there is a nonnegative constant vector $T=\left(t_{1}, t_{2}, \ldots, t_{n}\right)$ such that $v(T)=\sum_{i=1}^{n} a_{i} t_{i}$.

It is obvious that Butnariu game is an additive fuzzy capacity game. However, the fuzzy coalition $\widetilde{A}$ probably contains only some extremes or discrete points of the domain of $L(\widetilde{S})$, and $\widetilde{S} \subseteq L(N)$. Therefore, $v$ might be partially nonadditive or non-additive on its domains.

Let $s$ be a random variable; a subdecomposition of $s$ is a finite summation $\sum_{A_{i} \subseteq N} \alpha_{i} A_{i}$ that satisfies

$$
\sum_{A_{i} \subseteq N} \alpha_{i} 1_{A_{i}} \leq s \quad\left(\alpha_{i} \geq 0\right),
$$

where $1_{A_{i}}$ is an indicator of $A_{i} \subseteq N$.

A subdecomposition is a representation of a random variable as a positive linear combination of indicators.

Remark 19. For any random variable $s$, there are different subdecompositions of $s$. Moreover, for maximum decomposition of $s$, it may be different.

Example 20. Let players' set $N=\{1,2,3\}$; define a fuzzy coalition variable $s=(0.3,0.5,0.2)$ on $N$. For one subdecomposition $A_{1}=\{1,2\}$ and $A_{2}=\{2,3\}$. We take $\alpha_{1}=0.3$, $\alpha_{2}=0.2$, and then we get

$$
\sum_{A_{i} \subseteq N} \alpha_{i} A_{i}=0.3\{1,2\}+0.2\{2,3\}=(0.3,0.5,0.2)=s .
$$

Hence, the subdecomposition is a maximum decomposition of $s$.

On the other hand, if we consider other subdecompositions $A_{1}=\{2\}, A_{2}=\{1,2\}$, and $A_{3}=\{1,2,3\}$; take $\alpha_{1}=0.2$, $\alpha_{2}=0.1, \alpha_{3}=0.2$; and then

$$
\begin{aligned}
\sum_{A_{i} \subseteq N} \alpha_{i} A_{i} & =0.2\{2\}+0.1\{1,2\}+0.2\{1,2,3\} \\
& =(0.3,0.5,0.2)=s .
\end{aligned}
$$

We note that this subdecomposition is also a maximum decomposition of $s$ and $A_{1} \subseteq A_{2} \subseteq A_{3}$.

However, let $v(N)=10, v(\{1,2\})=v(\{1,3\})=5$, $v(\{2,3\})=9$ and $v(\{1\})=v(\{2\})=v(\{3\})=3$. 
Consiser

$$
\sum_{A_{i} \subseteq\{\{1,2\},\{2,3\}\}} \alpha_{i} v\left(A_{i}\right)=0.3 v(\{1,2\})+0.2 v(\{2,3\})=3.3 .
$$

Similarly,

$$
\begin{aligned}
\sum_{A_{i} \subseteq\{\{2\},\{1,2\},\{1,2,3\}\}} \alpha_{i} v\left(A_{i}\right)= & 0.2 v(\{2\})+0.1 v(\{1,2\}) \\
& +0.2 v(\{1,2,3\})=3.1
\end{aligned}
$$

It is not hard to find that the value of additive capacity on different maximum decomposition can be different.

Definition 21. Let $v$ be a capacity over $N$, and let $X$ be a nonnegative random variable; define the Concave integral as

$$
\int^{\mathrm{Cav}} X d v=\max \left\{\sum_{A \subseteq N} \alpha_{A} v(A), \sum_{A \subseteq N} \alpha_{A} 1_{A}=X, \alpha_{A} \geq 0\right\},
$$

where $1_{A}$ is an indicator of $A \subseteq N$.

Definition 22. Let $v$ be a fuzzy capacity game, and let $\widetilde{S} \in$ $L(N)$ be a random fuzzy coalition with nonnegative variable $s=\left(s_{1}, s_{2}, \ldots, s_{n}\right)$; define a game with Concave integral form $v^{\mathrm{Cav}}: L(N) \rightarrow R_{+} \cup\{0\}$ by

$$
v^{\mathrm{Cav}}(\widetilde{S})=\int^{\mathrm{Cav}} s d v=\min \{f(s)\}
$$

Let $1_{T}$ be an indicator of $T \subseteq N$ that

$$
1_{T}(U)= \begin{cases}1, & \text { if } T \subseteq U \\ 0, & \text { otherwise }\end{cases}
$$

And the minimum is taken all over concave and homogeneous functions $f: R_{+}^{n} \rightarrow R$ such that $f\left(1_{T}\right) \geq v(T)$ for every $T \subseteq N$.

By Definition 22, $v^{\mathrm{Cav}}(\widetilde{S})$ is based on decomposition of random variables of crisp game and can be gained by values on crisp coalitions which correspond to subdecompositions of $s$. We denote all fuzzy games defined by Concave integral as $\widetilde{G}^{\text {Cav }}$. It is easy to prove the following lemma.

Lemma 23. Let $v \in \widetilde{G}^{\text {Cav }}$ be a fuzzy capacity game, for every random fuzzy coalition $\widetilde{S} \in L(N)$ with nonnegative variable $s=\left(s_{1}, s_{2}, \ldots, s_{n}\right) ;$ then

$$
\int^{C a v} s d v=\max \left\{\sum_{T \subseteq N} \alpha_{T} v(T), \sum_{T \subseteq N} \alpha_{T} 1_{T}=s, \alpha_{T} \geq 0\right\} .
$$

The game $v^{\text {Cav }}: L(N) \rightarrow R_{+} \cup\{0\}$ can also be calculated by

$$
\begin{array}{ll}
v^{C a v} & (\widetilde{S})=\int^{C a v} s d v=\max \sum_{T_{i} \subseteq N} \alpha_{T_{i}} v\left(T_{i}\right) \\
\text { s.t } \quad & \sum_{T_{i} \subseteq N} \alpha_{T_{i}} 1_{T_{i}}=s, \\
& T_{i} \in[\widetilde{U}]_{\alpha_{T_{i}}}, \\
& v\left(T_{i}\right) \neq 0, \\
& \alpha_{T_{i}} \geq 0 .
\end{array}
$$

Remark 24. When $S \subseteq N$,

$$
\begin{array}{ll}
v(S) & =\int^{\mathrm{Cav}} 1_{S} d v=\max \sum_{T_{i} \subseteq N} \alpha_{T_{i}} v\left(T_{i}\right) \\
\text { s.t } & \sum_{T_{i} \subseteq N} \alpha_{T_{i}} 1_{T_{i}}=1, \\
& T_{i} \subseteq N, \\
& v\left(T_{i}\right) \neq 0, \\
& \alpha_{T_{i}}=1 .
\end{array}
$$

It can be easily seen that $\int^{\mathrm{Cav}} 1_{S} d v=$ $\max \left\{\sum_{T \subseteq N} v(T) \sum_{T \subseteq N} 1_{T}=1\right\}=v(S)$. It means that the fuzzy game with Concave integral extends crisp game.

Since crisp game $v\left(T_{i}\right), T_{i} \in[\widetilde{U}]_{\alpha_{T_{i}}}$ can be represented by simple unanimity games; it is easy to prove the following lemma.

Lemma 25. Let $v \in G^{C a v}(N)$ be a fuzzy capacity game, and let $\widetilde{S} \in L(N)$ be a random fuzzy coalition with nonnegative variable $s=\left(s_{1}, s_{2}, \ldots, s_{n}\right)$; then

$$
\begin{array}{ll}
v^{\text {Cav }}(\widetilde{S}) & =\int^{C a v} s d v=\max \sum_{T_{i} \subseteq N} \alpha_{T_{i}} \sum_{T \in P\left(T_{i}\right) \backslash\{\phi\}} c_{S}(v) u_{S} \\
\text { s.t. } & \sum_{T_{i} \subseteq N} \alpha_{T_{i}} 1_{T_{i}}=s, \\
& T_{i} \in[\widetilde{U}]_{\alpha_{T_{i}}}, \\
& v\left(T_{i}\right) \neq 0, \\
& \alpha_{T_{i}} \geq 0,
\end{array}
$$

where $c_{S}(v)=\sum_{T \in P\left(T_{i}\right): T \subseteq S}(-1)^{|S|-|T|} v(T)$.

Example 26. Let $N=\{1,2,3\}$ and let $v$ be a characteristic function on $N \cdot v(\{1\})=v(\{2\})=v(\{3\})=2, v(\{1,3\})=8$, $v(\{1,2\})=9, v(\{2,3\})=5$, and $v(\{1,2,3\})=10$. Let $S \in L(N)$ with the fuzzy coalition variable $s=(1,0.4,0.6)$. 
Suppose $v \in \widetilde{G}^{\mathrm{Cav}}$, we have

$$
\begin{array}{ll}
v^{\mathrm{Cav}} & (\widetilde{S})=\int^{\mathrm{Cav}} s d v=\max \sum_{T_{i} \subseteq N} \alpha_{T_{i}} v\left(T_{i}\right) \\
\text { s.t. } & \sum_{T_{i}} \alpha_{T_{i}} 1_{T_{i}}=(1,0.4,0.6), \\
& T_{i} \in\{\{1\},\{2\},\{3\},\{1,3\},\{1,2\},\{2,3\},\{1,2,3\}\}, \\
& \alpha_{T_{i}} \geq 0 .
\end{array}
$$

Then $T_{1}=\{1,2\}, T_{2}=\{1,3\}, \alpha_{T_{1}}=0.4$ and $\alpha_{T_{2}}=0.6$.

So $v^{\mathrm{Cav}}(\widetilde{S})=0.4 \times v(\{1,2\})+0.6 \times v(\{1,3\})=0.4 \times 9+$ $0.6 \times 8=8.4$.

If $v \in \widetilde{G}^{C}$, rearrange elements of $s$ as $0.4<0.6<1$; then by (11),

$$
\begin{aligned}
v^{\mathrm{Ch}}(\widetilde{S})= & \sum_{l=1}^{q(\widetilde{S})} v\left([\widetilde{S}]_{h_{l}}\right) \cdot\left(h_{l}-h_{l-1}\right) \\
= & v\left([\widetilde{S}]_{0.4}\right) \times 0.4+v\left([\widetilde{S}]_{0.6}\right) \\
& \times(0.6-0.4)+v\left([\widetilde{S}]_{1}\right) \times(1-0.6) \\
= & v(\{1,2,3\}) \times 0.4+v(\{1,3\}) \times 0.2+v(\{1\}) \times 0.4 \\
= & 6.4 .
\end{aligned}
$$

We note that $v^{\mathrm{Cav}}(\widetilde{S}) \geq v^{\mathrm{Ch}}(\widetilde{S})$.

Example 27. Consider again Example 5, for the fuzzy coalition $\widetilde{U}$, by inequality (28), we have

$$
\begin{array}{cl}
v^{\mathrm{Cav}}(\widetilde{U})=\int^{\mathrm{Cav}} u d v=\max \sum_{T_{i} \subseteq N} \alpha_{T_{i}} v\left(T_{i}\right) \\
\text { s.t. } \quad \sum_{T_{i}} \alpha_{T_{i}} 1_{T_{i}}=(0.4,0.4,0.6,0.7), \\
T_{i} \in P(N), \\
v\left(T_{i}\right) \neq 0, \\
\alpha_{T_{i}} \geq 0 .
\end{array}
$$

Hence

$$
\begin{gathered}
T_{1}=\{1,2,3,4\}, \quad T_{2}=\{3,4\}, \quad T_{3}=\{4\}, \\
\alpha_{T_{1}}=0.4, \quad \alpha_{T_{2}}=0.2, \quad \alpha_{T_{3}}=0.1, \\
v^{\text {Cav }}(\widetilde{S})=\max \sum_{T_{i} \subseteq N} \alpha_{T_{i}} v\left(T_{i}\right) \\
=0.4 \times v(\{1,2,3,4\})+0.2 v(\{3,4\}) \\
+0.1 v(\{4\})=61.6 .
\end{gathered}
$$

Thus, $v^{\mathrm{Cav}}(\widetilde{S})=v^{\mathrm{Ch}}(\widetilde{S})$.
We know that $v^{\mathrm{Cav}}(\widetilde{\mathrm{S}})=\int^{\mathrm{Cav}} s d v$ is the maximum of the values $\sum_{i=1}^{k} \alpha_{T_{i}} \mu\left(T_{i}\right)$ among all possible decompositions of $\widetilde{S}$ with the coalition variable $s$.

In the fuzzy game given by Tsurumi et al., the Choquet integral of nonnegative $h$ with respect to a capacity $v$ is defined by $\int^{\mathrm{Ch}} H d v=\sum_{l=1}^{q(\widetilde{S})}\left(h_{l}-h_{l-1}\right) \cdot v\left([\widetilde{S}]_{h_{l}}\right)$,

$$
\begin{aligned}
\int^{\mathrm{Ch}} H d v & =\sum_{i=1}^{n}\left(H_{\sigma(i)}-H_{\sigma(i-1)}\right) v\left(\sigma_{(i)}, \sigma_{\sigma(i+1)}, \ldots, \sigma_{(n)}\right) \\
& =\sum_{l=1}^{q(\widetilde{S})}\left(h_{l}-h_{l-1}\right) \cdot v\left([\widetilde{S}]_{h_{l}}\right)
\end{aligned}
$$

where $H$ is the set of all rates of participation such that $0=$ $h_{0}<h_{1}<h_{2}<\cdots<h_{q(\widetilde{S})}$ and $\sigma_{(\bullet)}$ is a permutation of $N$.

Let $\alpha_{i}=h_{i}-h_{i-1}$; note that $H=\sum \alpha_{i} 1_{[\widetilde{S}]_{h_{i}}}$ is a decomposition of $H$. That is, the Choquet integral is defined under the special decomposition of $H$. By contrast, all possible decompositions are allowed in the Concave integral. In this way, it implies that $\int^{\mathrm{Ch}} H d v \leq \int^{\mathrm{Cav}} H d v$.

Lemma 28. Let $v \in G^{\text {Cav }}(N)$ be a fuzzy capacity game, and let $\widetilde{S} \in L(N)$ be a random fuzzy coalition with nonnegative variable $s=\left(s_{1}, s_{2}, \ldots, s_{n}\right)$; then

$$
v^{C h}(\widetilde{S})=\int^{C h} s d v=\max \sum_{T_{l} \subseteq N} \alpha_{T_{l}} v\left(T_{l}\right),
$$

where $T_{i} \supseteq T_{j}(\forall i<j)$.

Proof. Let $v \in G^{\mathrm{Cav}}(N)$ be a fuzzy capacity game, $\widetilde{S} \in L(N)$ and $s=\left(s_{1}, s_{2}, \ldots, s_{n}\right)$; by Definition 8 , we get

$$
v(\widetilde{S})=\int^{C h} s d v=\sum_{l=1}^{q(\widetilde{S})} v\left([\widetilde{S}]_{h_{l}}\right) \cdot\left(h_{l}-h_{l-1}\right),
$$

where $h_{1}<h_{2}<\cdots<h_{q(\widetilde{S})}$. Take $T_{l}=[\widetilde{S}]_{h_{l}}$ and $\alpha_{T_{l}}=h_{l}-h_{l-1}$, since $[\widetilde{S}]_{h_{1}} \supseteq[\widetilde{S}]_{h_{2}} \supseteq \cdots \supseteq[\widetilde{S}]_{h_{q(\widetilde{S})}}, T_{i} \supseteq T_{j}(\forall i<j)$.

On the other hand, by $v \in G^{\mathrm{Cav}}(N),[\widetilde{S}]_{h_{0}},[\widetilde{S}]_{h_{1}}, \ldots,[\widetilde{S}]_{h_{q(\tilde{S})}}$ is a maximum decomposition with the fuzzy coalition variable $\sum_{T_{l} \subseteq N} \alpha_{T_{l}} 1_{T_{l}}=\sum_{T_{l} \subseteq N}\left(h_{l}-h_{l-1}\right) 1_{T_{l}}=\left(h_{1}-h_{0}, \ldots, h_{l}-\right.$ $\left.h_{l-1}, \ldots, h_{q(\widetilde{S})}-h_{q(\widetilde{S})-1}\right)$.

$$
\text { So } \sum_{l=1}^{q(\widetilde{S})} v\left([\widetilde{S}]_{h_{l}}\right) \cdot\left(h_{l}-h_{l-1}\right)=\max \sum_{T_{l} \subseteq N} \alpha_{T_{l}} v\left(T_{l}\right) \text {. }
$$

Therefore, when $v \in G^{\mathrm{Cav}}(N)$, then $v^{\mathrm{Ch}}(\widetilde{S})=\int^{\mathrm{Ch}} s d v=$ $\max \sum_{T_{l} \subseteq N} \alpha_{T_{l}} v\left(T_{l}\right)$.

For any two nonnegative random fuzzy coalitions $\widetilde{U}$ and $\widetilde{S}$ with variables $u$ and $s$, define distance on $L(N)$ by $d(\widetilde{S}, \widetilde{U})=$ $\max _{i \in N}\left|s_{i}-u_{i}\right|$.

Theorem 29. Let $v \in G^{\text {Cav }}(N)$ be a fuzzy capacity game, then $v$ is continuous with respect to fuzzy coalition variables. 
Proof. Let $\widetilde{U}$ and $\widetilde{S}$ be any two fuzzy coalitions and nonnegative variables $u$ and $s$; we will prove that if $d(\widetilde{S}, \widetilde{U}) \rightarrow 0$, then $d\left(v^{\mathrm{Cav}}(\widetilde{S}), v^{\mathrm{Cav}}(\widetilde{U})\right) \rightarrow 0$. By the definitions,

$$
\begin{aligned}
& v^{\mathrm{Cav}}(\widetilde{U})=\int^{\mathrm{Cav}} u d v=\max \sum_{T_{i} \subseteq N} \alpha_{U_{i}} v\left(U_{i}\right) \\
& \text { s.t. } \sum_{U_{i} \subseteq N} \alpha_{U_{i}} 1_{U_{i}}=u, \\
& U_{i} \in[\widetilde{U}]_{\alpha_{U_{i}}}, \\
& v\left(U_{i}\right) \neq 0, \\
& \alpha_{U_{i}} \geq 0 \text {, } \\
& v^{\mathrm{Cav}}(\widetilde{S})=\int^{\mathrm{Cav}} s d v=\max \sum_{S_{i} \subseteq N} \alpha_{S_{i}} v\left(S_{i}\right) \\
& \text { s.t. } \sum_{T_{i} \subseteq N} \alpha_{S_{i}} 1_{S_{i}}=s, \\
& S_{i} \in[\widetilde{U}]_{\alpha_{S_{i}}}, \\
& v\left(S_{i}\right) \neq 0, \\
& \alpha_{S_{i}} \geq 0, \\
& \left|v^{\mathrm{Cav}}(\widetilde{S})-v^{\mathrm{Cav}}(\widetilde{U})\right| \\
& =\left|\max \sum_{S_{i} \subseteq N, v\left(S_{i}\right) \neq 0} \alpha_{S_{i}} v\left(S_{i}\right)-\max \sum_{U_{i} \subseteq N, v\left(U_{i}\right) \neq 0} \alpha_{U_{i}} v\left(U_{i}\right)\right| \\
& \leq \max \left|\sum_{S_{i} \subseteq N, v\left(S_{i}\right) \neq 0} \alpha_{S_{i}} v\left(S_{i}\right)-\sum_{U_{i} \subseteq N, v\left(U_{i}\right) \neq 0} \alpha_{U_{i}} v\left(U_{i}\right)\right| \\
& \leq_{S_{i}, U_{i} \subseteq N, v\left(S_{i}\right) \text { or } v\left(U_{i}\right) \neq 0} \sum\left|\alpha_{S_{i}} v\left(S_{i}\right)-\alpha_{U_{i}} v\left(U_{i}\right)\right| \\
& \leq \sum_{S_{i}, U_{i} \subseteq N, v\left(S_{i}\right) \text { or } v\left(U_{i}\right) \neq 0}\left(v\left(S_{i}\right), v\left(U_{i}\right)\right)\left|\alpha_{S_{i}}-\alpha_{U_{i}}\right| \\
& \leq \sum \max _{S_{i} \subseteq N, U_{i} \subseteq N}\left(v\left(S_{i}\right), v\left(U_{i}\right)\right) \max _{i \in N}\left|s_{i}-u_{i}\right| \\
& \leq d(\widetilde{S}, \widetilde{T}) \sum_{S_{i} \subseteq N, U_{i} \subseteq N}\left(v\left(S_{i}\right), v\left(U_{i}\right)\right) .
\end{aligned}
$$

Note that $v \in G(N, v)$ is finite such that $\sum \max _{S_{i} \subseteq N, U_{i} \subseteq N}\left(v\left(S_{i}\right)\right.$, $\left.v\left(U_{i}\right)\right) \leq M$ and $M>0$ is a sufficient great constant, that is,

$$
0 \leq d\left(v^{\mathrm{Cav}}(\widetilde{S}), v^{\mathrm{Cav}}(\widetilde{U})\right) \leq \operatorname{Md}(\widetilde{S}, \widetilde{U})
$$

Therefore, $v \in G^{\mathrm{Cav}}(N)$ is continuous with respect to fuzzy coalition variables.
Example 30. Continue with Example 26, for another fuzzy coalition $\widetilde{U}$ with $u=(0.4,1,0.6)$; then by inequality (28), we have that

$$
\begin{aligned}
v^{\mathrm{Cav}}(\widetilde{U}) & =\int^{\mathrm{Cav}} u d v=\max \sum_{T_{i} \leq N} \alpha_{T_{i}} v\left(T_{i}\right) \\
& =0.4 v(\{1,2\})+0.6 v(\{2,3\})=6.6 .
\end{aligned}
$$

By (11), we also get

$$
\begin{aligned}
v^{\mathrm{Ch}}(\widetilde{U})= & \int^{\mathrm{Ch}} u d v=\sum_{l=1}^{q(\widetilde{U})} v\left([\widetilde{U}]_{h_{l}}\right) \cdot\left(h_{l}-h_{l-1}\right) \\
= & v\left([\widetilde{U}]_{0.4}\right) \times 0.4+v\left([\widetilde{U}]_{0.6}\right) \times(0.6-0.4) \\
& +v\left([\widetilde{U}]_{1}\right) \times(1-0.6) \\
= & 0.4 v(\{1,2,3\})+0.2 v(\{2,3\})+0.4 v(\{2\})=5.8 .
\end{aligned}
$$

From the above examples, we note that $v^{\mathrm{Cav}}(\widetilde{S}) \geq v^{\mathrm{Cav}}(\widetilde{U})$ while $v^{\mathrm{Ch}}(\widetilde{S}) \geq v^{\mathrm{Ch}}(\widetilde{U})$.

Remark 31. When $v$ is convex, $v^{\mathrm{Cav}}(\widetilde{S})=v^{\mathrm{Ch}}(\widetilde{S})$ and $v^{\mathrm{Cav}}(\widetilde{U})=$ $v^{\mathrm{Ch}}(\widetilde{U})$; it is apparent $v^{\mathrm{Cav}}(\widetilde{S}) \geq v^{\mathrm{Cav}}(\widetilde{U}) \Leftrightarrow v^{\mathrm{Ch}}(\widetilde{S}) \geq v^{\mathrm{Ch}}(\widetilde{U})$.

Theorem 32. Let $v \in G(N, \widetilde{v})$ be a fuzzy capacity game; if $v$ is convex, then $v^{\mathrm{Cav}}(\widetilde{S}) \geq v^{\mathrm{Cav}}(\widetilde{U})$ if and only if $v^{\mathrm{Ch}}(\widetilde{S}) \geq v^{\mathrm{Ch}}(\widetilde{U})$ for any $\widetilde{S}, \widetilde{U} \in L(N)$.

\section{Imputation of Fuzzy Games with the Concave Integral}

Imputation is an important concept; players will get rational share from a cooperative team work, where he gets more shares than he gets by working alone, and the sum of all players' shares in a team is equal to the team value. Next, we will extend the crisp imputation to fuzzy game.

Definition 33. Let $v \in G^{\mathrm{Cav}}(N)$; a fuzzy coalition $\widetilde{U} \in L(N)$ with variable $u=\left(u_{1}, u_{2}, \ldots, u_{n}\right)$; a vector function $x^{\mathrm{Cav}}$ : $L(u) \rightarrow R_{+}^{n}$ is called an imputation if

$$
\begin{aligned}
& \text { (1) } x_{i}^{\mathrm{Cav}}(\widetilde{U})=0, \forall i \notin \operatorname{Supp}(\widetilde{U}), \\
& \text { (2) } \sum_{i \in N} x_{i}^{\mathrm{Cav}}(\widetilde{U})=v^{\mathrm{Cav}}(\widetilde{U}), \\
& \text { (3) } x_{i}^{\mathrm{Cav}}(\widetilde{U}) \geq v^{\mathrm{Cav}}\left(\widetilde{e_{i}}\right),
\end{aligned}
$$

where $\widetilde{e}_{i}=\left(0,0, \ldots, u_{i}, \ldots, 0\right)$ and $x^{\mathrm{Cav}}(\widetilde{U})=\left(x_{1}^{\mathrm{Cav}}(\widetilde{U})\right.$, $\left.x_{2}^{\mathrm{Cav}}(\widetilde{U}), \ldots, x_{n}^{\mathrm{Cav}}(\widetilde{U})\right)$.

We denote the set of all imputations of the fuzzy game $v \in G^{\mathrm{Cav}}(N)$ on the fuzzy coalition $\widetilde{U} \in L(N)$ as $I\left(v^{\mathrm{Cav}}\right)(\widetilde{U})$.

Theorem 34. Let a vector $x=\left(x_{1}, x_{2}, \ldots, x_{n}\right) \in I(v)(N)$ for crisp cooperative game $v \in G(N, v)$, and then $x^{\text {Cav }}=$ $\left(x_{1}^{\mathrm{Cav}}, x_{2}^{\mathrm{Cav}}, \ldots, x_{n}^{\mathrm{Cav}}\right) \in I\left(v^{\mathrm{Cav}}\right)(\widetilde{U})$ is an imputation for game $v(\widetilde{U}) \in G^{\text {Cav }}(N)$ given by Definition 21, where $\sum_{T \subseteq N} \alpha_{T} 1_{T}=$ $u\left(\forall \alpha_{T} \geq 0\right)$ and $x_{i}^{C a v}=\max \left\{\sum_{T \subseteq \operatorname{supp}(\widetilde{U})} \alpha_{T} x_{i}(T)\right\}$. 
Proof. For crisp cooperative game $v \in G(N, v)$, define fuzzy coalition $\widetilde{U} \in L(N)$ by $\sum_{T \subseteq N} \alpha_{T} 1_{T}=u\left(\forall \alpha_{T} \geq 0\right)$. For $T \subseteq$ $\operatorname{supp}(\widetilde{U})$, if $i \notin \operatorname{Supp}(\widetilde{U})$, then $x_{i}(T)=0$. So $x_{i}^{\mathrm{Cav}}(\widetilde{U})=0$, $\forall i \notin \operatorname{Supp}(\widetilde{U})$.

Since $x=\left(x_{1}, x_{2}, \ldots, x_{n}\right) \in I(v)(N)$, we have

$$
\begin{aligned}
\sum_{i \in N} x_{i}^{\mathrm{Cav}}= & \sum_{i \in N} \max \sum_{T \subseteq N} \alpha_{T} x_{i}(T) \\
= & \sum_{i \in N} \max \sum_{T \subseteq N \backslash \operatorname{supp}(\widetilde{U})} \alpha_{T} x_{i}(T) \\
& +\sum_{i \in N} \max \sum_{T \subseteq \operatorname{supp}(\widetilde{U})} \alpha_{T} x_{i}(T) \\
= & \max \sum_{i \in N} \sum_{T \subseteq \operatorname{supp}(\widetilde{U})} \alpha_{T} x_{i}(T) \\
= & \max \sum_{T \subseteq \operatorname{supp}(\widetilde{U})} \alpha_{T} \sum_{i \in N} x_{i}(T) \\
= & \max \sum_{T \subseteq \operatorname{supp}(\widetilde{U})} \alpha_{T} v(T) \\
= & v^{\operatorname{Cav}}(\widetilde{U}) .
\end{aligned}
$$

On the other hand,

$$
\begin{aligned}
x_{i}^{\mathrm{Cav}}(\widetilde{U}) & =\max \sum_{T \subseteq \operatorname{Supp}(\widetilde{U})} \alpha_{T} x_{i}(T) \\
& =\max \sum_{T \subseteq \operatorname{Supp}(\widetilde{U})} \alpha_{T} x_{i}(T)+\max \sum_{T \subseteq N \backslash \operatorname{Supp}(\widetilde{U})} \alpha_{T} x_{i}(T) \\
& =\max \sum_{T \subseteq N} \alpha_{T} x_{i}(T) \\
& \geq \max \sum_{T \subseteq N, i \in T} \alpha_{T} v(\{i\}) \\
& =v^{\operatorname{Cav}}\left(\widetilde{e}_{i}\right) .
\end{aligned}
$$

So $x_{i}^{\mathrm{Cav}}(\widetilde{U}) \geq v^{\mathrm{Cav}}\left(\widetilde{e}_{i}\right)$.

Therefore, $x^{\mathrm{Cav}}=\left(x_{1}^{\mathrm{Cav}}, x_{2}^{\mathrm{Cav}}, \ldots, x_{n}^{\mathrm{Cav}}\right) \in I\left(v^{\mathrm{Cav}}\right)(\widetilde{U})$.

Corollary 35. Let a vector $x=\left(x_{1}, x_{2}, \ldots, x_{n}\right) \in I(v)(N)$ for crisp cooperative game $v \in G(N, v)$, and fuzzy coalition $\widetilde{U} \in$ $L(N)$ has fuzzy coalition variable $\sum_{T \subseteq N} \alpha_{T} 1_{T}=u\left(\forall \alpha_{T} \geq 0\right)$, then $y \in I\left(v^{\mathrm{Cav}}\right)(\widetilde{U})$ can be calculated by

$$
y=\int^{C a v} u d x
$$

Remark 36. When $v \in G(N, v)$ and $I(v)(N)$ is nonempty, then $I\left(v^{\mathrm{Cav}}\right)(\widetilde{U})$ is nonempty for fuzzy game $v(\widetilde{U}) \in G^{\mathrm{Cav}}(N)$, where fuzzy coalition $\widetilde{U}$ is determined by $\sum_{T \subseteq N} \alpha_{T} 1_{T}=$ $u\left(\forall \alpha_{T} \geq 0\right)$.
TABle 2: The crisp Shapley values.

\begin{tabular}{lccccccc}
\hline$S$ & $\{1\}$ & $\{2\}$ & $\{3\}$ & $\{1,2\}$ & $\{1,3\}$ & $\{2,3\}$ & $\{1,2,3\}$ \\
\hline $\mathrm{Sh}_{1}(v)$ & 100 & 0 & 0 & 200 & 200 & 0 & 300 \\
$\mathrm{Sh}_{2}(v)$ & 0 & 200 & 0 & 250 & 0 & 400 & 450 \\
$\mathrm{Sh}_{3}(v)$ & 0 & 0 & 200 & 0 & 250 & 400 & 450 \\
\hline
\end{tabular}

Corollary 37. Let $v \in G_{0}(N, v)$; fuzzy coalition $\widetilde{U}$ is determined by $\sum_{T \subseteq N} \alpha_{T} 1_{T}=u\left(\forall \alpha_{T} \geq 0\right)$, and then

$$
z=\int^{C a v} u d \operatorname{Sh}(v) \in I\left(v^{C a v}\right)(\widetilde{U}),
$$

where $\operatorname{Sh}(v)=\left(\operatorname{Sh}_{1}(v), \operatorname{Sh}_{2}(v), \ldots, S h_{n}(v)\right)$ is the Shapley vector for crisp game $v \in G_{0}(N, v)$.

Example 38. Let $N=\{1,2,3\}, v(\{1\})=100, v(\{2\})=$ $v(\{3\})=200, v(\{1,2\})=v(\{1,3\})=600, v(\{2,3\})=800$, and $v(\{1,2,3\})=1200$.

Suppose a decomposition $T_{1}=\{1,2,3\}, T_{2}=\{2,3\}, T_{3}=$ $\{3\}, \alpha_{T_{1}}=0.2, \alpha_{T_{2}}=0.2$, and $\alpha_{T_{3}}=0.1$.

Then the fuzzy coalition $\widetilde{U}$ defined by $\sum_{T \subseteq N} \alpha_{T} 1_{T}=$ $(0.2,0.4,0.5)$.

Consider

$$
\begin{aligned}
v^{\mathrm{Cav}}(\widetilde{U}) & =\int^{\mathrm{Cav}} u d v=\max \sum_{T_{i} \subseteq N} \alpha_{T_{i}} v\left(T_{i}\right) \\
& =0.2 v(\{1,2,3\})+0.2 v(\{2,3\})+0.1 v(\{3\})=420 .
\end{aligned}
$$

The crisp Shapley values are obtained as in Table 2.

$$
\begin{aligned}
\sum_{T \subseteq \operatorname{supp}(\widetilde{U})} \alpha_{T} x_{i}(T)= & \alpha_{T_{1}} x\left(T_{1}\right)+\alpha_{T_{2}} x\left(T_{2}\right)+\alpha_{T_{3}} x\left(T_{3}\right) \\
= & 0.2(300,450,450)+0.2(0,400,400) \\
& +0.1(0,0,200) \\
= & (60,170,190) .
\end{aligned}
$$

In fact, $\sum_{i \in n} \sum_{T \subseteq \operatorname{supp}(\widetilde{U})} \alpha_{T} x_{i}(T)=420=v^{\mathrm{Cav}}(\widetilde{U})$, so $(60,170,190) \in I\left(v^{\mathrm{Cav}}\right)(\widetilde{U})$.

Let us consider an another decomposition $T_{1}=\{1,2,3\}$, $T_{2}=\{2,3\}, T_{3}=\{3\}, \alpha_{T_{1}}^{\prime}=0.1, \alpha_{T_{2}}^{\prime}=0.3$ and $\alpha_{T_{3}}^{\prime}=0.1$.

Note that $\sum_{T \subseteq N} \alpha_{T}^{\prime} 1_{T}=(0.2,0.4,0.5)$, so these two decomposition have the same coalition variable

$$
\begin{aligned}
\sum_{T \subseteq \operatorname{supp}(\widetilde{U})} \alpha_{T_{i}}^{\prime} x_{i}(T)= & \alpha_{T_{1}}^{\prime} x\left(T_{1}\right)+\alpha_{T_{2}}^{\prime} x\left(T_{2}\right)+\alpha_{T_{3}}^{\prime} x\left(T_{3}\right) \\
= & 0.1(300,450,450)+0.3(0,400,400) \\
& +0.1(0,0,200) \\
= & (30,165,185),
\end{aligned}
$$




$$
\begin{aligned}
\sum_{T_{i} \subseteq N} \alpha_{T_{i}} v\left(T_{i}\right) & =0.1 v(\{1,2,3\})+0.3 v(\{2,3\})+0.1 v(\{3\}) \\
& =380 \leq v^{\mathrm{Cav}}(\widetilde{U}) .
\end{aligned}
$$

In addition, $z=\int^{\mathrm{Cav}} u d \operatorname{Sh}(v)=\alpha_{T_{1}} \operatorname{Sh}\left(T_{1}\right)+\alpha_{T_{2}} \operatorname{Sh}\left(T_{2}\right)+$ $\alpha_{T_{3}} \operatorname{Sh}\left(T_{3}\right)=(60,170,190)=x^{\mathrm{Cav}}(\widetilde{U})$.

\section{Conclusions}

In this paper, an extended game with fuzzy coalition variables and a characteristic function calculation technique is presented for solving a class of games when the available information is limited to a few events. The linear formula of the new game is established by the maximum integral on these characteristic functions on subdecompositions of crisp coalitions.

By comparing the present definitions for games with fuzzy coalitions which are actually defined by different integrals, a new integral expression of fuzzy cooperative game is discussed in detail from the view of Concave integral. Because the characteristic function of the proposed game considers all events or possible coalitions, it may be greater than that of game defined by Choquet integral, which was only calculated on nest coalitions.

It is natural that the Concave integral representation for fuzzy game can be regarded as a general form of cooperative game with fuzzy coalitions.

\section{Conflict of Interests}

The authors declare that they do not have commercial or associative interests that represent a conflict of interests in connection with this paper. There are no professional or other personal interests that can inappropriately influence their submitted work.

\section{Acknowledgments}

This research was supported by the National Natural Science Foundation, China (Grant nos. 71271029, and 71371030) and the Education of Humanities and Social Science Research on Self-fund Project, China (Grant no. 11YJE630003). The authors would like to express their deep gratitude to the referee for his/her valuable comments and suggestions.

\section{References}

[1] D. Granot, "Cooperative games in stochastic characteristic function form," Management Science, vol. 23, no. 6, pp. 621-630, 1977.

[2] F. R. Fernández, J. Puerto, and M. J. Zafra, "Cores of stochastic cooperative games with stochastic orders," International Game Theory Review, vol. 4, no. 3, pp. 265-280, 2002.

[3] L. A. Zadeh, "Fuzzy sets," Information and Computation, vol. 8, no. 3, pp. 338-353, 1965.
[4] M. Mareš, "Weak arithmetics of fuzzy numbers," Fuzzy Sets and Systems, vol. 91, no. 2, pp. 143-153, 1997.

[5] D. Dubois and H. Prade, Fundamentals of Fuzzy Sets, The Handbooks of Fuzzy Stes, Kluver Academic, Boston, Mass, USA, 2000.

[6] J. P. Aubin, "Coeur et valeur des jeux flous à paiements latéraux," Comptes Rendus Hebdomadaires Des Séances De 1'Académie Des Sciences A, vol. 279, pp. 891-894, 1974.

[7] R. Branzei, D. Dimitrov, and S. Tijs, Models in Cooperative Game Theory: Crisp, Fuzzy and Multichoice Games, Springer, Berlin, Germany, 2005.

[8] D. Butnariu, "Fuzzy games: a description of the concept," Fuzzy Sets and Systems, vol. 1, no. 3, pp. 181-192, 1978.

[9] M. Tsurumi, T. Tanino, and M. Inuiguchi, "A Shapley function on a class of cooperative fuzzy games," European Journal of Operational Research, vol. 129, no. 3, pp. 596-618, 2001.

[10] S. Borkotokey, "Cooperative games with fuzzy coalitions and fuzzy characteristic functions," Fuzzy Sets and Systems, vol. 159, no. 2, pp. 138-151, 2008.

[11] E. Lehrer, "A new integral for capacities," Economic Theory, vol. 39, no. 1, pp. 157-176, 2009.

[12] M. Sugeno and T. Murofushi, "Choquet's integral as an integral form for a general class of fuzzy measures," in Proceedings of the 2nd IFSA Congress, vol. 1, pp. 408-411, 1987.

[13] Y. Azrieli and E. Lehrer, "Extendable cooperative games," Journal of Public Econ Theory, vol. 9, no. 6, pp. 1069-1078, 2007.

[14] D. Butnariu, "Stability and Shapley value for an $n$-persons fuzzy game," Fuzzy Sets and Systems, vol. 4, no. 1, pp. 63-72, 1980.

[15] D. Butnariu and T. Kroupa, "Shapley mappings and the cumulative value for $n$-person games with fuzzy coalitions," European Journal of Operational Research, vol. 186, no. 1, pp. 288-299, 2008. 


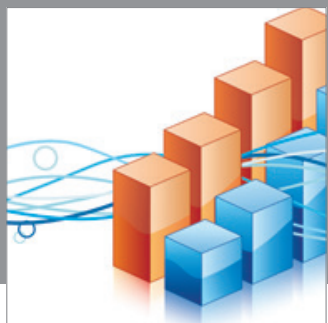

Advances in

Operations Research

mansans

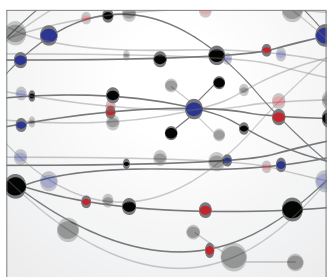

The Scientific World Journal
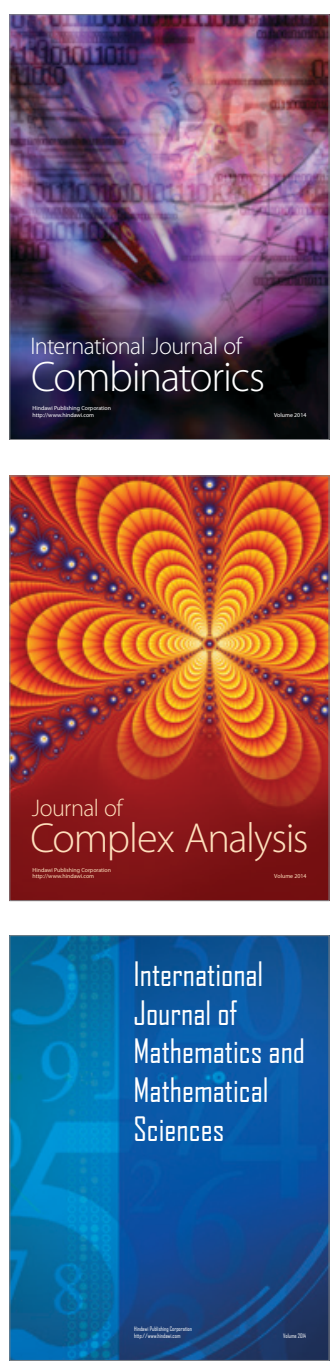
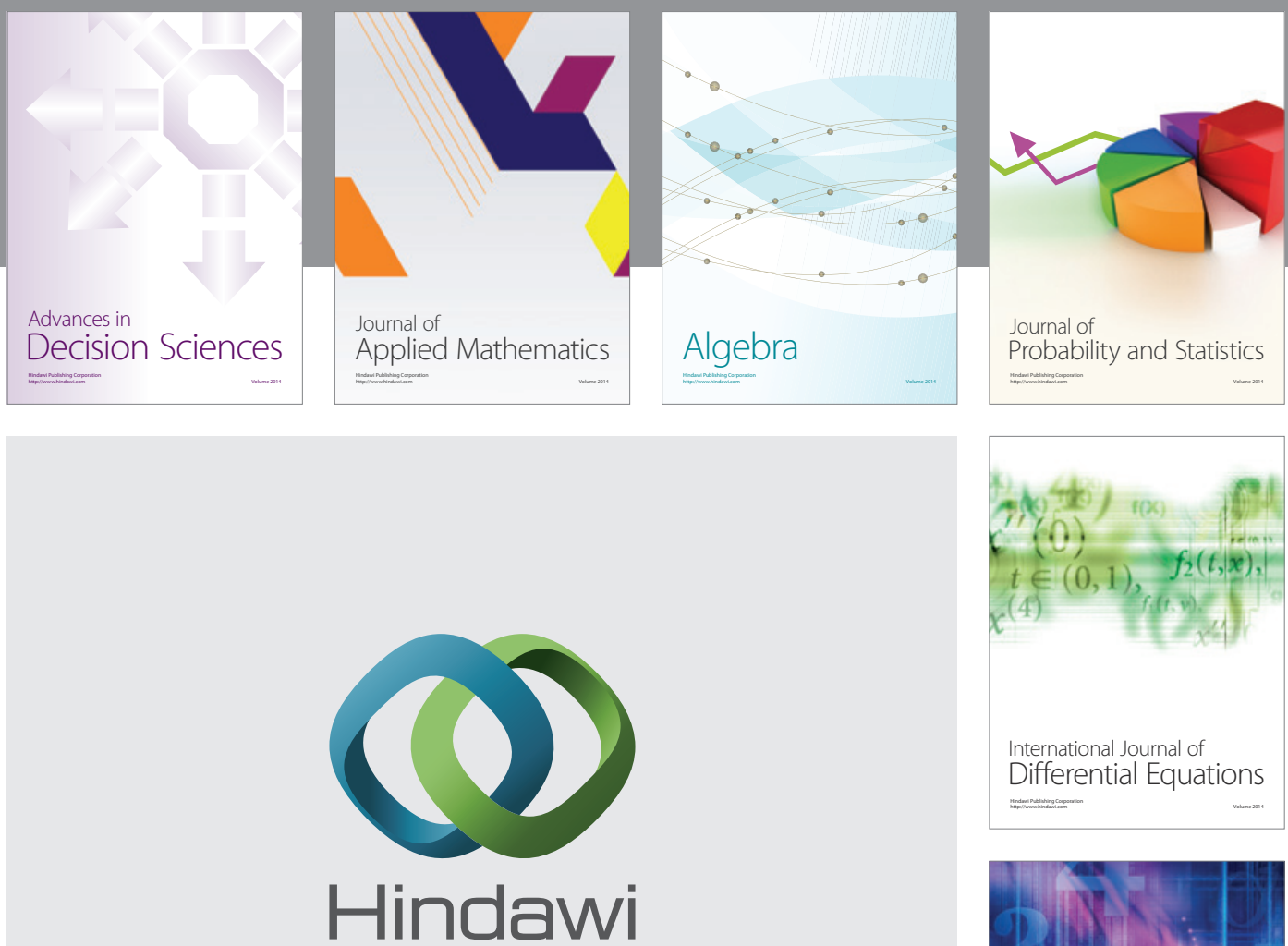

Submit your manuscripts at http://www.hindawi.com
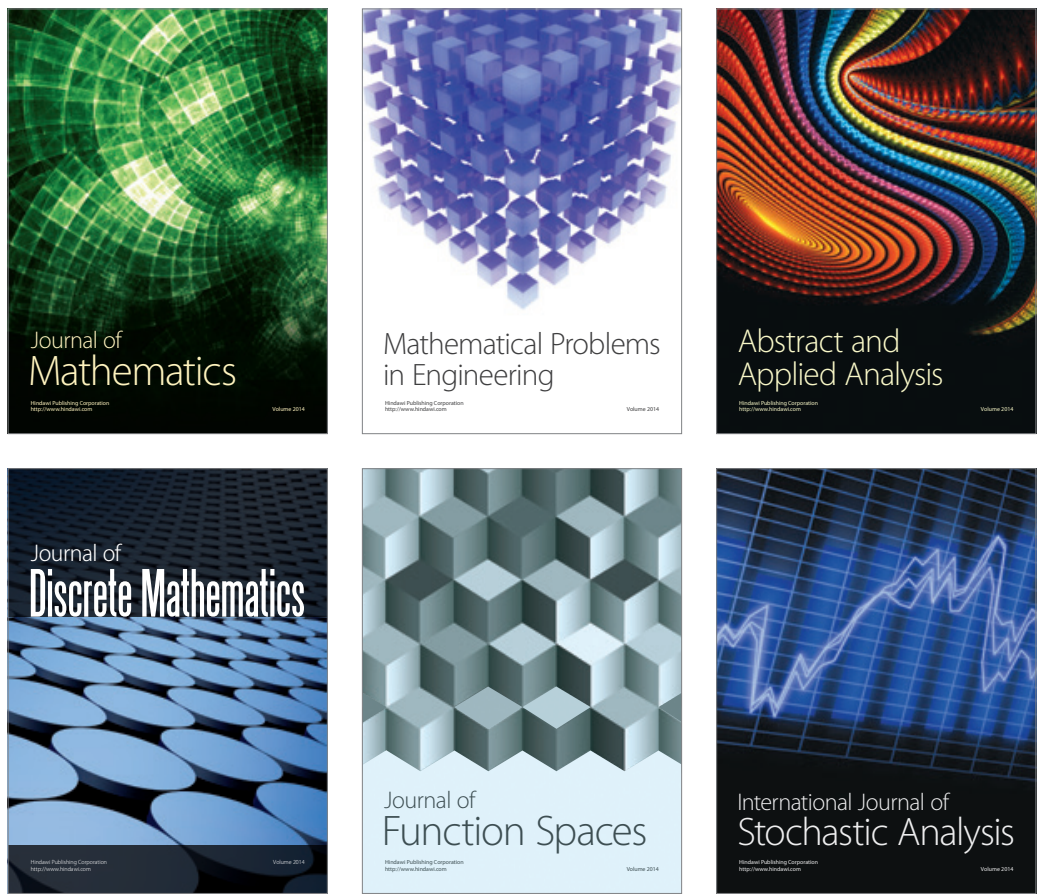

Journal of

Function Spaces

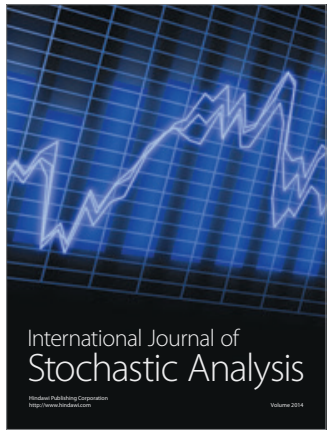

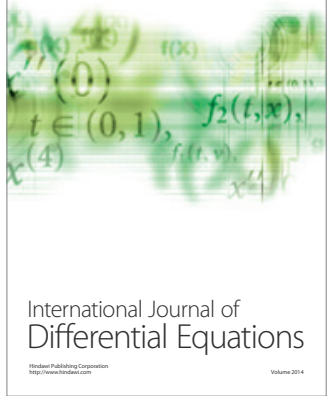
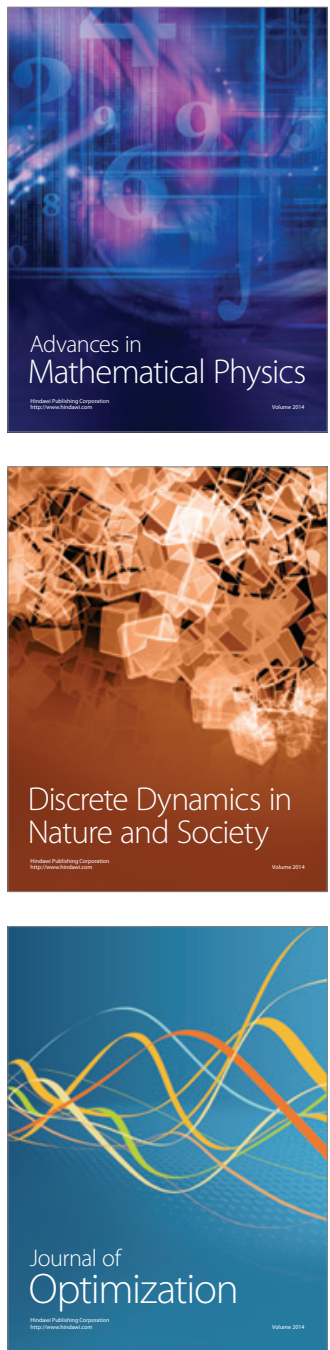